\title{
Comparison of Strategies for Coupled Flow-Thermal Analysis of Thermal Protection System at Hypersonic Flight Condition
}

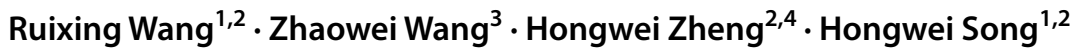

Received: 6 May 2019 / Revised: 24 August 2019 / Accepted: 18 September 2019

(c) The Korean Society for Aeronautical \& Space Sciences 2019

\begin{abstract}
Coupled flow-thermal analysis is crucial for the performance evaluation and structural design of hypersonic vehicles. In this study, several strategies for computing the coupled flow-thermal response of air-breathing hypersonic flights in practical engineering are developed and compared. First, the basic direct correction method that amends wall heat flux via recovery temperature and wall temperature is proposed to approximate flow-thermal effects efficiently. Second, the improved DCM (IDCM) is further developed by interpolating the cold wall heat flux and the recovery temperature between adjacent trajectory points to improve computational accuracy. Third, the iteration solution method (ISM) that obtains solutions through mass and energy balances at a common interface by iterations between CFD analysis code and CSD analysis code is also presented. Thermal response and flow characteristics are compared through a test case of an air-breathing hypersonic vehicle. The results show that the thermal response tendencies are consistent by DCMs and ISM. However, for DCMs, the impact of hot wall on the flow characteristics is ignored, whereas it is fully considered in ISM; thus, the thickened boundary flow and complicated internal flow can be captured. However, while comparing computational efficiency, DCMs have a prominent advantage over ISM due to the decoupling algorithm and parallel strategy. Based on this, in the actual design process of an air-breathing hypersonic vehicle, the designers can select the proper flow-thermal analysis method according to the different design stages.
\end{abstract}

Keywords Coupled flow-thermal analysis · Thermal protection system · Basic direct correction method (BDCM) · Improved DCM (IDCM) · Iteration solution method (ISM)

\section{Introduction}

One of the main challenges for hypersonic vehicles is the severe aerodynamic heating upon the surfaces during service. In this case, a thermal protection system (TPS) is designed to serve as a shield that is responsible for protecting

Ruixing Wang

wangruixing@imech.ac.cn

1 Key Laboratory for Mechanics in Fluid Solid Coupling Systems, Institute of Mechanics, Chinese Academy of Sciences, Beijing 100190, China

2 School of Engineering Science, University of Chinese Academy of Science, Beijing 100049, China

3 Research and Development Center, China Academy of Launch Vehicle Technology, Beijing 100076, China

4 Key Laboratory of High Temperature Gas Dynamics, Institute of Mechanics, Chinese Academy of Sciences, Beijing 100190, China the inner structure of a hypersonic vehicle from extreme temperature $[9,18,25]$. In hypersonic flow environments, TPS materials conduct heat and participate in radiative energy exchange [20, 32, 34]. Thus, the design and analysis of TPS for hypersonic vehicles in general requires computations involving the transient thermal response of material thermophysics, and a sequence of steady-state flow to determine the history of aerodynamic heating. This problem is of critical importance, since any improvement in the computation fidelity may lead to reduction in TPS weight, which significantly reduces the cost and improves the performance of hypersonic vehicle [29, 35, 37].

For the coupled flow-thermal analysis of hypersonic vehicles, one of the most essential aspects is the coupling effect between the aerodynamic heat and the wall temperature on the fluid-solid interface. Neglecting of the coupling effect leads to a prominent error since the interaction effect between the surface heat flux and the wall temperature is indeed strong when the wall temperature is high. In the 
earliest literature, the coupling problem was handled by using approximations derived from the hypersonic boundary layer theory for the fluid dynamics and one-dimensional in-depth heat conduction for the material thermal response $[2,11,13]$. In addition, only simple geometric shapes such as flat plates, spheres, and sphere cones can be applied in this situation.

With improvements in the computational algorithms, many flow-thermal coupled analysis approaches have been presented and developed in recent years. Conti et al. [5] coupled Navier-Stokes calculations to the material thermal response for axisymmetric re-entry flow fields, in which the surface temperature was computed as a result of the interface surface energy balance. Chen and Henline [3] derived a general surface boundary condition for the Navier-Stokes equations, and the coupled flow-thermal analysis was conducted in a loose way. Later, an iteration method that aimed to substantially reduce the number of iterations for a converged solution was proposed [4, 22, 23]. The flow response code and thermal response code are loosely coupled in such a manner that the aerodynamic heat computations use surface temperatures obtained from the thermal response code and the thermal response computations use the surface energy-balance conditions as well as the convective heat-transfer coefficients obtained from flow solver. Ferrero and D'Ambrosio [7] presented a two-dimensional finite volume heat conduction solver coupled with a hypersonic fluid solver to solve the CHT problem over a body immersed in a high-speed flow. Zhang et al. [36] described a novel, time-adaptive, loosely coupled analysis strategy for efficiently predicting the conjugate heat transfer problems in hypersonic flows, in which an adaptive coupling time step size approach based on a control theory point of view was proposed and embedded into this strategy. Rahaim et al. [27] developed a coupled finite volume method and dual reciprocity boundary element method to solve the transient flow-thermal problem in the supersonic regime. Meng et al. [21] proposed a new algorithm of the global tightly coupled transient heat transfer based on the quasi-steady flow field for solving the long-term conjugate heat transfer (CHT) problem.

Additionally, with advances in the computer hardware, numerous successful applications of flow-thermal coupled analysis methods have been reported in practical engineering systems. Kuntz et al. [16, 17] and Hassan et al. [12] presented an iterative, loosely coupled approach between a CFD code and a material thermal response code, and then attempted to predict the ablation phenomena on a hypersonic reentry vehicle moving along a ballistic trajectory. Kontinos [15] presented a two-dimensional boundary element method and then loosely coupled it to a hypersonic CFD algorithm to solve the flow-thermal coupled problem over metallic thermal protection panels at the leading edge of the X-33 in a hypersonic flow. Tabiei and Sockalingam [30] developed a procedure for modeling a thermal protection system for the IRV-2 reentry vehicle by constructing a multiphysics framework for hypersonic reentry vehicles. The computational fluid dynamics code (FLUENT) and the material thermal and structural response code (LS-DYNA) are loosely coupled to achieve the solution. Zhao et al. [38] proposed a coupling methodology to solve simultaneously the external flow field and the resultant heating on the structure for a generic missile nose cone, in which two flow solvers were employed to solve the hypersonic flowfield and the very lowspeed flowfield, respectively. Guo et al. [10] and Qin et al. [26] presented a loosely coupled fluid-thermal analysis to illustrate the thermal response of different configurations and the relevant flow field variation for a spiked blunt body flying at hypersonic speeds.

In summary, a number of efforts have been made to solve the coupled flow-thermal problems for hypersonic vehicles. Nevertheless, according to the current research state described above, there are still some issues that need to be further investigated.

1. To the author's knowledge, the coupled flow-thermal problem for the air-breathing hypersonic vehicle [28] in practical engineering has been rarely investigated until now, while most existing studies have focused on cases with either a two-dimensional model or a simple threedimensional model. An air-breathing hypersonic vehicle at hypersonic speed could lead to significant thermal interactions between the external flowfield, internal flowfield and internal structure, these, indeed, deserve more attention in future investigations.

2. According to the review of related literatures, until now, most published literatures have focused on promoting the iteration-based flow-thermal coupled strategies, and the research concerning the efficient engineering approximation method is rare. However, it should be noted that different analysis method should be adopted according to the design stage of the hypersonic vehicle. This means that an efficient engineering approximation method is suitable in the conceptual design stage in which the shape and structure form of the flight keeps updating, whereas an iteration method is required in the detailed design stage to accurately describe the coupled flow-thermal effect. Therefore, the future research on establishing the coupled flow-thermal analysis methodology containing both engineering approximate method and the iterative coupled method is needed.

Under this circumstance, two kinds of coupled flow-thermal analysis strategies, namely, the engineering approximate method (the direct correction method) and the loosely coupled method (the iteration-based method) are proposed in this 
paper to support the entire design process of air-breathing hypersonic vehicles. Besides, the strategies of the two coupled flow-thermal analyses for the thermal protection systems of the hypersonic vehicle are compared to determine the advantages and disadvantages of each method. The remainder of the paper is structured as follows: the specific description of two typical strategies for coupled flow-thermal analysis is presented in Sect. 2. The particular contents of the computational fluid dynamics (CFD) analytical model and the finite element method (FEM) analytical model are presented in Sect. 3. The results and discussions are stated in Sect. 4. The conclusions are derived at the end of the paper.

\section{Outline of Two Coupled Flow-Thermal Analytical Strategies}

\subsection{Coupled Analysis Based on Direct Correction Methods (DCM)}

\subsubsection{Basic DCM Approach}

To adopt the coupled flow-thermal analysis in engineering practice, the basic direct correction method (BDCM), which amends the wall heat flux via recovery temperature and wall temperature, is developed to improve the analysis efficiency.

According to aerothermodynamics, the wall heat flux $q_{\mathrm{w}}$ is proportional to the difference between the recovery temperature and wall temperature, i.e.

$q_{\mathrm{w}}=\alpha\left(T_{\mathrm{r}}-T_{\mathrm{w}}\right)$,

where $T_{\mathrm{r}}$ and $T_{\mathrm{w}}$ denote the recovery temperature and wall temperature, respectively. $\alpha$ is the surface heat transfer coefficient, which is related to several physical quantities, such as flight altitude, Mach number, and skin temperature. The dimensionless surface heat transfer coefficient, i.e., Stanton number $S t$, is more commonly used

$S t=\alpha /\left(c_{\mathrm{p}} \rho u\right)$,

where $c_{\mathrm{p}}$ is the specific heat at constant pressure, $\rho$ is the density, and $u$ is the flow velocity.

Combining Eqs. (1) and (2), $q_{\mathrm{w}}$ can be rewritten as

$q_{\mathrm{w}}=\operatorname{St\rho uc}_{\mathrm{p}}\left(T_{\mathrm{r}}-T_{\mathrm{w}}\right)$.

Considering that $c_{\mathrm{p}}$ varies inconspicuously at different wall temperatures when the other flight conditions, including the flight height and flight speed, are the same, $c_{\mathrm{p}}$ is assumed to be constant in engineering to improve the computational efficiency. Thus, the wall heat flux under the condition of constant wall temperature $T_{0}$, can be expressed as

$q_{T=T_{0}}=\operatorname{St\rho uc}_{\mathrm{p}}\left(T_{\mathrm{r}}-T_{0}\right)$.
Then, the real wall heat flux can be approximated by integrating Eqs. (3) and (4):

$q_{\mathrm{w}}=q_{T=T_{0}} \frac{T_{\mathrm{r}}-T_{\mathrm{w}}}{T_{\mathrm{r}}-T_{0}}$.

For the TPS in hypersonic vehicles, the fluid is coupled to the thermal conduction through the surface energy balance, given by

$q_{\mathrm{n}}=q_{\mathrm{w}}-q_{\mathrm{rad}}$,

where $q_{\mathrm{n}}$ is the heat flux due to conduction, and $q_{\mathrm{rad}}$ is the heat flux due to radiation.

According to the theory of heat radiation,

$q_{\mathrm{rad}}=\sigma \varepsilon\left(T_{w}^{4}-T_{0}^{4}\right)$.

Here, $\sigma$ represents the Stefan-Boltzmann constant is equal to $5.67 \mathrm{e}-8 \mathrm{~W} /\left(\mathrm{m}^{-2} \mathrm{~K}^{-4}\right)$; and $\varepsilon$ represents the emissivity, which is related to the structural material property.

Then, substituting Eqs. (5) and (7) into Eq. (6), $q_{\mathrm{n}}$ can be rewritten as

$q_{\mathrm{n}}=q_{T=T_{0}} \frac{T_{\mathrm{r}}-T_{\mathrm{w}}}{T_{\mathrm{r}}-T_{0}}-\sigma \varepsilon\left(T_{\mathrm{w}}^{4}-T_{0}^{4}\right)$.

In particular, according to the temperature boundary layer theory, $T_{\mathrm{r}}$ can be calculated by

$T_{\mathrm{r}}=T_{\infty}\left(1+r \frac{\gamma-1}{2} M a_{\infty}^{2}\right)$,

where $T_{\infty}$ is the inflow static temperature; $M a_{\infty}$ is the inflow Mach number; $\gamma$ is the ratio of specific heat, which is usually set as 1.4; and $r$ is the recovery temperature coefficient, which is usually set as 0.85 for laminar flow and 0.90 for turbulent flow [14].

Overall, the heat flux due to conduction can be expressed as

$q_{\mathrm{n}}=q_{T=T_{0}} \frac{T_{\infty}\left(1+r \frac{\gamma-1}{2} M a_{\infty}^{2}\right)-T_{\mathrm{w}}}{T_{\infty}\left(1+r \frac{\gamma-1}{2} M a_{\infty}^{2}\right)-T_{0}}-\sigma \varepsilon\left(T_{\mathrm{w}}^{4}-T_{0}^{4}\right)$,

where $q_{T=T_{0}}$ can be calculated by CFD analysis, $T_{\mathrm{w}}$ can be obtained using FEM analysis, and the other parameters can be easily determined from the freestream characteristics and structural material properties.

Considering that the characteristic time scale for hypersonic flows to reach the steady state is much smaller than that of the transient structural heat transfer problem, the assumption that the hypersonic flow behavior can reach the steady state instantaneously compared to the relative low evolution of the heat transfer in the solid can be introduced [19]. Moreover, Giles [8] concluded that numerical stability could be improved by passing temperature from the solid to 
the fluid while transferring heat flux from the fluid to the solid. Therefore, based on the abovementioned concepts, an efficient engineering analysis strategy, namely, BDCM, for the coupled flow-thermal problem of air-breathing hypersonic vehicle in hypersonic flows is developed as show in Fig. 1. Further, the implementation strategy of the BDCM approach can be described as follows.

1. For a trajectory involving $n$ flight conditions, the time points can be denoted as $\left[0, t_{1}, t_{2}, \ldots, t_{n-1}\right]$. At the initial time $t=0$, a certain uniform temperature distribution is induced in the solid depending on the initial condition, and then the surface temperature $T_{0}$ is transferred onto the fluid coupling surface as a temperature boundary condition.

2. Steady hypersonic flow simulations of all the discrete flight conditions are conducted using CFD code with the wall temperature boundary set to be a uniform value $T_{0}$, which is also termed the cold wall boundary. In this way, the wall heat flux under the cold wall condition can be obtained and denoted as $\left[q_{T=T_{0}}^{t=0}, q_{T=T_{0}}^{t=t_{1}}, q_{T=T_{0}}^{t=t_{2}}, \ldots\right.$, $\left.q_{T=T_{0}}^{t=t_{n-1}}\right]$.

3. Transient thermal analysis of the TPS from time level 0 to $t_{1}$ is conducted using FEM code. Considering that the flow field can be assumed to be transient stable, the recovery temperature $T_{\mathrm{r}}$ and the heat flux under the cold wall situation $q_{T=T_{0}}$ between the adjacent trajectory points are supposed to be constant. Therefore, the heat flux due to conduction $q_{\mathrm{n}}$ at any time step between 0 and $t_{1}$ can be directly modified by Eq. (8). To improve the computational efficiency, the step size of the transient thermal analysis based on FEM code, which is denoted as $\Delta t_{\mathrm{s}}$, is set to be adaptive.

4. The heat transfer simulations from time level $t_{i}$ to $t_{i+1}$ $(i=1,2, \ldots,(n-2))$ can be accomplished in the same manner until the transient thermal analysis of the entire trajectory is completed.

\subsubsection{Improved DCM Approach}

In practical engineering projects, as shown in Fig. 2, the following situations are commonly encountered: (1) The time interval between the adjacent trajectory points may be large in a practical engineering project owning to the limited information; (2) The flight condition changes dramatically between the adjacent trajectory points; (3) Situation (1) and situation (2) occur simultaneously.

In such a case, the analysis error would increase if the recovery temperature $T_{\mathrm{r}}$ and the heat flux with the cold wall $q_{T=T_{0}}$ are considered constant during the adjacent trajectory points. Consequently, in this study, BDCM is further developed by interpolating $T_{\mathrm{r}}$ and $q_{T=T_{0}}$ between the adjacent trajectory points as displayed in Fig. 2. In this manner, the processes involving continuous changes in both the cold wall heat flux and the recovery temperature are considered to some extent, which reflects the actual

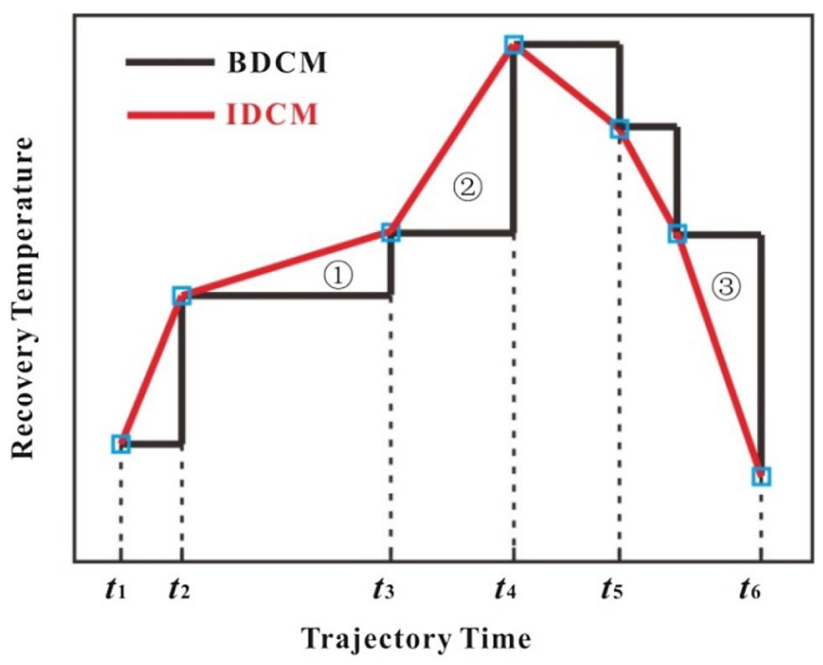

Fig. 2 The treatment of recovery temperature in BDCM and IDCM
Fig. 1 Flow-thermal engineering approximating procedure based on BDCM

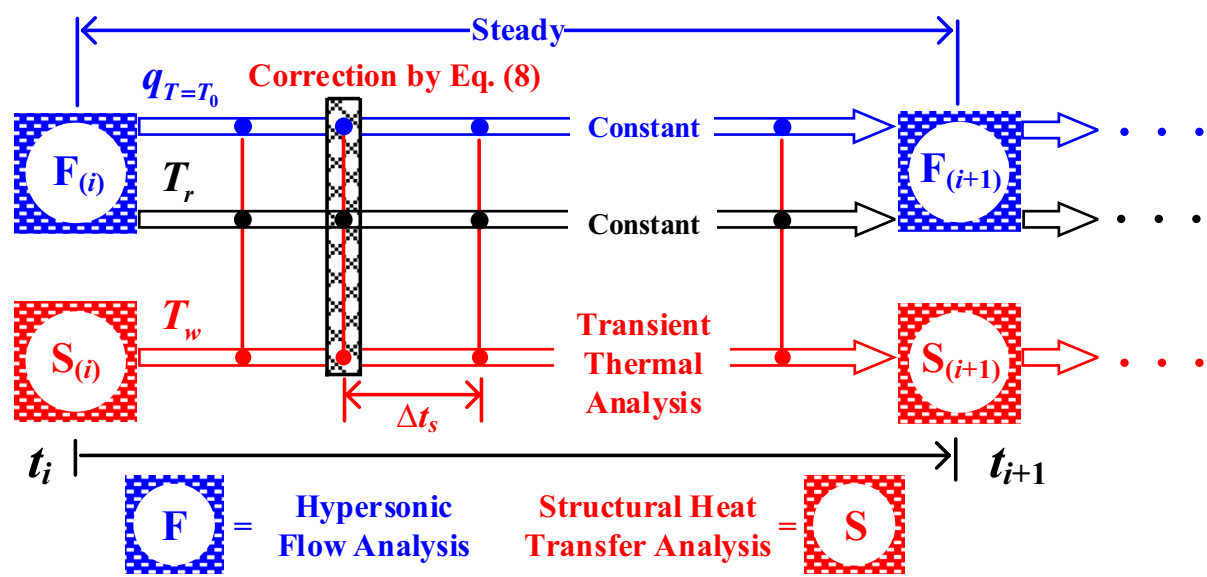


flight situations more reasonably. In particular, in the improved DCM approach (IDCM), the heat flux due to conduction $q_{\mathrm{n}}$ is also corrected and updated with an adaptive time step according to the specified characteristic step in the solid heat conduction analysis. The implementation process can be illustrated in Fig. 3.

\subsection{Coupled Analysis Based on the Iteration Solution Method (ISM)}

Unlike DCMs that require only one iteration by the correction strategy, ISM obtains the coupling solutions through several iterations between a finite element code and a finite volume flow code. As described in Fig. 4, the analyses of
Fig. 3 Flow-thermal engineering approximating procedure based on IDCM
Fig. 4 Flow-thermal loose coupling procedure based on ISM
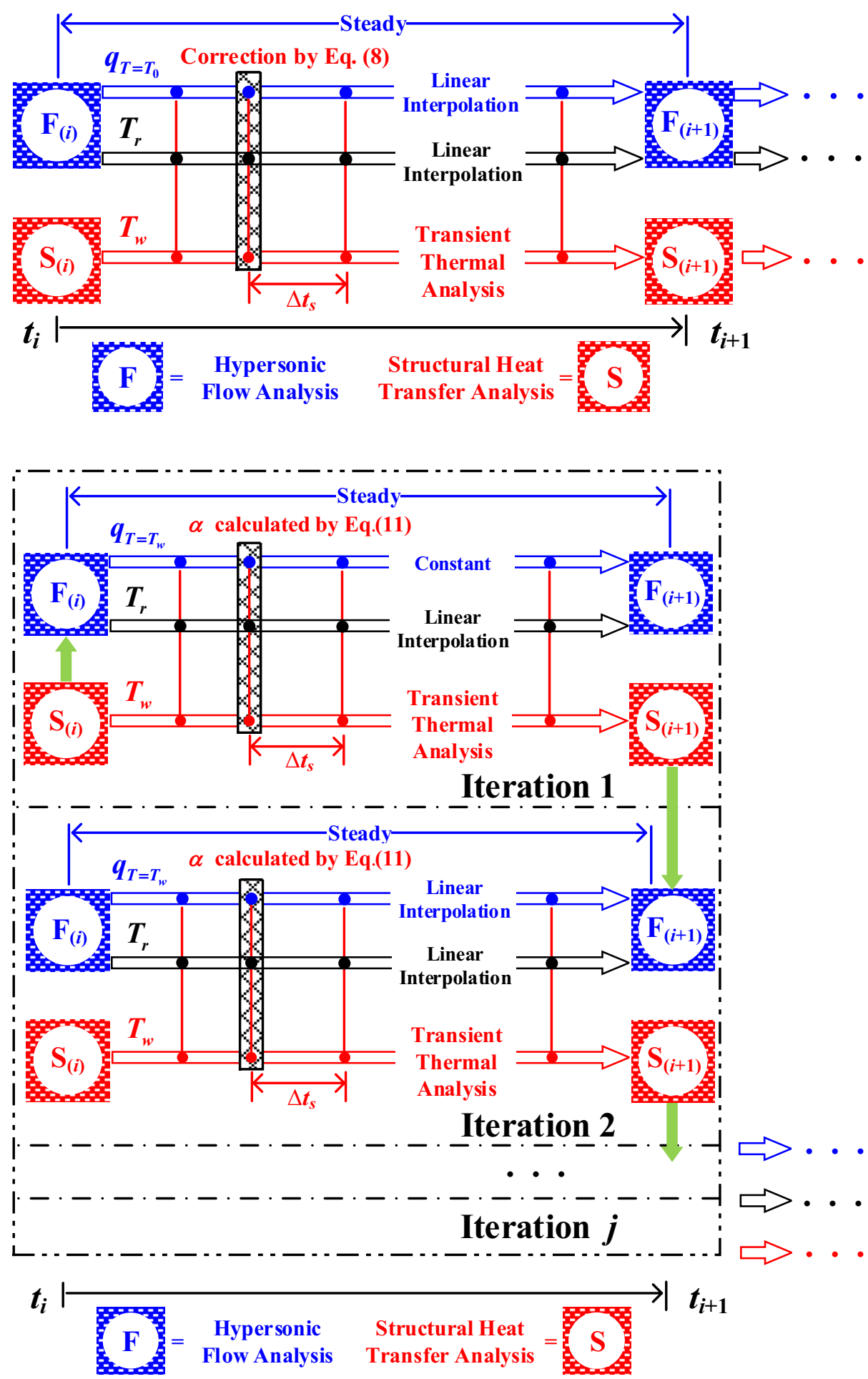
CFD and FEM are sequentially conducted in ISM and the executing process can be detailed as follows.

1. The CFD analysis is conducted for the trajectory point $i$ under the wall temperature condition, i.e., the hot wall condition, which can be obtained in the previous cycle. In particular, the wall temperature for the initial trajectory point is set as the uniform value of the ambient temperature.

2. It is concluded that the convective conditions can significantly improve the convergence efficiency and greatly highly reduce the number of iterations [3, 4]. Thus, in this step, the surface heat flux is converted into the surface heat transfer coefficient using

$\alpha=q_{\mathrm{w}} /\left(T_{\mathrm{r}}-T_{\mathrm{w}}\right)$.

3. The recovery temperature and surface heat transfer coefficient are applied on the FEA model as the heat transfer boundary condition and then the transient heat transfer analysis is conducted. Thus, the temperature field is obtained.

4. The CFD analysis is carried out for the trajectory point $(n+1)$ under the wall temperature distribution obtained in step [3]. Moreover, the surface heat transfer coefficient for the trajectory point $(n+1)$ can be then acquired.

5. Considering that the recovery temperature and the exchange coefficient change significantly between two adjacent trajectory points, the interpolation strategy is also utilized, and the step size of modification is set as the specified characteristic step in the solid heat conduction analysis. The heat transfer between trajectory points $n$ and $(n+1)$ is therefore recalculated under the heat transfer boundary condition.

6. Step 5 is repeated until convergence is achieved, and a reasonable temperature field between trajectory points $n$ and $(n+1)$ is also obtained.

Specially, two things should be noted here: First, the convergence criterion is constructed to stop the inner iterations adaptively to keep the computation time as low as possible. If $\mathbf{T}^{i}$ and $\mathbf{T}^{i+1}$ denote the temperature distribution for the inner iteration $i$ and $i+1$ respectively, the convergence criterion is defined as the relative difference between two successive iterations in the maximum norm, and the inner loop is considered to be converged when the relative difference belongs to $[0,1]$, which can be expressed as follows:

$0 \leq \frac{\left\|\mathbf{T}^{i}-\mathbf{T}^{i+1}\right\|_{\infty}}{\left\|\mathbf{T}^{i}\right\|_{\infty}+\left\|\mathbf{T}^{i+1}\right\|_{\infty}} \leq 1$.

When Eq. (12) is satisfied, the coupled quantity values remain more or less constant between consecutive data exchanges and the coupled solution cannot be improved by continuing the inner iterations, then the simulation can proceed to the next time step.

Second, since multiple CFD analyses between the trajectory points $n$ and $(n+1)$ are computationally expensive, an adjustable computational strategy is established to improve the computational efficiency. The strategy can be conducted as follows: for the first CFD analysis, the computation is performed as usual; for the rest CFD analyses, the initial condition is set as the results obtained in the previous CFD analysis. As the flow field characteristics become closer along with iteration times, the computation time taken in the CFD analysis can be remarkably reduced.

\section{Computational Model}

\subsection{CFD Model}

\subsubsection{In-House CFD Code Polysim}

In this paper, aerodynamic heat analysis is conducted using the in-house code Polysim [39-41], which is a cell-centered finite volume solver based on a hybrid mesh. The code is developed based on the unstructured grid data architecture to ensure flexibilities of handling complex geometries. Unlike finite difference, the finite volume formulation discretizes the integral governing equations on arbitrary-shaped control volumes. The control volume in Polysim can be any of the following types: tetrahedron, hexahedron, pyramid and prism. Instead of the simple average method for the gradients of variables at each face, the volume average is applied. In addition, an improved Green-Gauss method for the calculation of the gradient at the cell centroid is developed. These two techniques for the discretization of the viscous flux terms improve both the accuracy and robustness of the code. The aerodynamic performance obtained using this in-house code Polysim has been examined by several widely used benchmark test cases. It has been shown that the numerical results agree well with experimental data [39-41].

\subsubsection{Governing Equations}

Polysim is a cell-centered finite volume solver which solves the Favre-averaged Navier-Stokes equations

$\Gamma^{-1} \frac{\mathrm{d}}{\mathrm{d} t} \int_{\Omega} W \mathrm{~d} \Omega+\int_{\partial \Omega} F(U) \mathrm{d} A=0$,

and the turbulence equation

$\frac{\partial \Xi}{\partial t}+\nabla \cdot(\Xi u)=-\nabla \cdot D+P-\varepsilon$,

with the turbulence variables 


$$
\Xi=\left(\begin{array}{c}
k \\
\omega
\end{array}\right)
$$

In Eq. (12), $\Gamma$ is the preconditioned matrix [33], $W$ is the vector of primitive variables, $U$ is the vector of conservative variables, and $F$ is the flux vector

$\Gamma=\left[\begin{array}{ccccc}\rho_{\mathrm{P}}^{\mathrm{M}} & 0 & 0 & 0 & \rho_{\mathrm{T}} \\ \mu_{1} \rho_{\mathrm{P}}^{\mathrm{M}} & \rho & 0 & 0 & \rho_{\mathrm{T}} u_{1} \\ \mu_{2} \rho_{\mathrm{P}}^{\mathrm{M}} & 0 & \rho & 0 & \rho_{\mathrm{T}} u_{2} \\ \mu_{3} \rho_{\mathrm{P}}^{\mathrm{M}} & 0 & 0 & \rho & \rho_{\mathrm{T}} u_{3} \\ H \rho_{\mathrm{P}}^{\mathrm{M}}-\delta & \rho u_{1} & \rho u_{2} & \rho u_{3} & \rho_{\mathrm{T}} H+\rho c_{\mathrm{P}}\end{array}\right]$.

And

$$
\begin{aligned}
W & =\left(\begin{array}{c}
p \\
\vec{u} \\
T
\end{array}\right), \quad U=\left(\begin{array}{c}
\rho \\
\rho \vec{u} \\
E
\end{array}\right), \quad F=F^{\mathrm{c}}-F^{v}, \\
F^{\mathrm{c}} & =\left(\begin{array}{c}
\rho \vec{u} \\
\rho \vec{u} \otimes \vec{u}+P[\mathrm{I}] \\
(E+P) \vec{u}
\end{array}\right), \quad F^{v}=\left(\begin{array}{c}
0 \\
{[\tau]} \\
([\tau] \cdot \vec{u})+\vec{q}
\end{array}\right) .
\end{aligned}
$$

Here, $\rho$ is the density, $\vec{u}$ is the velocity, $E$ is the total energy, $P$ is the pressure, and $[\tau]$ is the stress tensor,

$[\tau]=\left(\mu_{\mathrm{L}}+\mu_{\mathrm{t}}\right)\left[\nabla \vec{u}+\nabla^{\mathrm{T}} \vec{u}-\frac{2}{3}(\nabla \cdot \vec{u})[I]\right]$

\subsubsection{Numerical Discretization}

Equation (12) is discretized by using the point-implicit algorithm,

$$
\begin{aligned}
D^{m-1} \Delta W_{\mathrm{i}}^{\mathrm{m}}= & -\left(\mathfrak{R}_{i}^{m-1}+\sum_{j \in \text { neigbor }}\left[F_{\mathrm{f}}\left(W_{i}^{m-1}, W_{j}^{m}, \vec{n}\right)\right.\right. \\
& \left.\left.-F_{\mathrm{f}}\left(W_{i}^{m-1}, W_{j}^{m-1}, \vec{n}\right)\right] \cdot A_{\mathrm{f}}\right),
\end{aligned}
$$

with $\Delta W_{\mathrm{i}}^{\mathrm{m}}=W_{i}^{m+1}-W_{i}^{m}$.

Here, $D$ is the diagonal matrix and the residue is defined as

$\Re_{i}^{m-1}=\sum_{\mathrm{f}} F_{\mathrm{f}}\left(U^{-}, U^{+}, \vec{n}\right) \cdot A_{\mathrm{f}}$,

with $F_{\mathrm{f}}\left(U^{-}, U^{+}, \vec{n}\right)$ is the approximated numerical flux of the face $f$ and $A_{\mathrm{f}}$ denotes the area of the face $f$. In this study, the low diffusion kinetic scheme is employed to calculate the convective numerical flux

$F_{\mathrm{f}}^{\mathrm{c}}=(1-\alpha) F_{\mathrm{n}}\left(Q_{\mathrm{f}}\right)+\alpha F_{\mathrm{n}}^{\mathrm{KFVS}}$, where the fluxes are expressed as

$$
\begin{aligned}
& F_{\mathrm{n}}=\left(\begin{array}{c}
\rho u_{\mathrm{n}} \\
\rho \mathbf{u} u_{\mathrm{n}}+p \mathbf{n} \\
\rho H u_{\mathrm{n}}
\end{array}\right)=F_{\mathrm{n}}^{+}+F_{\mathrm{n}}^{-}, \\
& F_{\mathrm{n}}^{ \pm}=\left(\begin{array}{c}
\rho\left\langle\xi_{\mathrm{n}}^{1}\right\rangle^{ \pm} \\
\rho \mathbf{u}\left\langle\xi_{\mathrm{n}}^{1}\right\rangle^{ \pm}+p \mathbf{n}\left\langle\xi_{\mathrm{n}}^{0}\right\rangle^{ \pm} \\
\left(\rho E+\frac{1}{2} p\right)\left\langle\xi_{\mathrm{n}}^{1}\right\rangle^{ \pm}+\frac{1}{2} p u_{\mathrm{n}}\left\langle\xi_{\mathrm{n}}^{0}\right\rangle^{ \pm}
\end{array}\right) .
\end{aligned}
$$

Here, $\alpha$ represents the artificial viscosity and the conservative variables $Q_{\mathrm{f}}$ can be obtained by using the same splitting rule as that for the flux.

To calculate the viscous flux, the flow variables and their gradients on faces must be also calculated. Because we use the cell-centered method, and all the flow variables are stored at the cell centers, the estimation of the variables on faces must be constructed as accurately as possible. The integrals on the faces require the best estimation of all the flow variables on these faces. In the present code, a volume weighted average of the adjacent cell gradients is employed

$(\vec{\nabla} W)_{\mathrm{f}}=\frac{\Omega_{\mathrm{L}} \vec{\nabla} W_{\mathrm{L}}+\Omega_{\mathrm{R}} \vec{\nabla} W_{\mathrm{R}}}{\Omega_{\mathrm{L}}+\Omega_{\mathrm{R}}}$.

The gradient in the cell center is calculated by the Green-Gauss method:

$(\vec{\nabla} W)_{\mathrm{c}}=\frac{1}{\Omega_{\mathrm{c}}} \sum_{\mathrm{f}}\left(W_{\mathrm{f}} \vec{n}_{\mathrm{f}}\right) A_{\mathrm{f}}$.

The face state in this calculation employs a new inverse distance weighted average rather than the simple average:

$W_{\mathrm{f}}=\frac{\Omega_{\mathrm{Rf}} W_{\mathrm{L}}+\Omega_{\mathrm{Lf}} W_{\mathrm{R}}}{\Omega_{\mathrm{Lf}}+\Omega_{\mathrm{Rf}}}$.

\subsubsection{CFD Model for Hypersonic Flight}

The geometry considered in this work is a typical X-51Alike air-breathing hypersonic vehicle. In the CFD analytical model, the CFD computational grid is a structured grid created in Pointwise Version 18.0 [24] and the flow field is constrained to four boundary conditions, namely, hypersonic far field, hypersonic outlet, symmetry on the centerline and no slip wall, as illustrated in Fig. 5a.

Additionally, it is well acknowledged that the first cell normal spacing near the wall is important for the aerodynamic heating numerical simulation, thus the impact study of the first cell spacing near the wall on the surface flux is conducted. The freestream and flight conditions of 


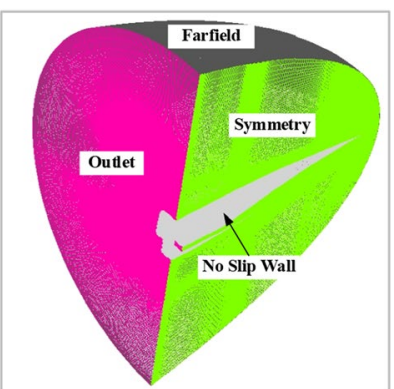

(a) Boundary condition

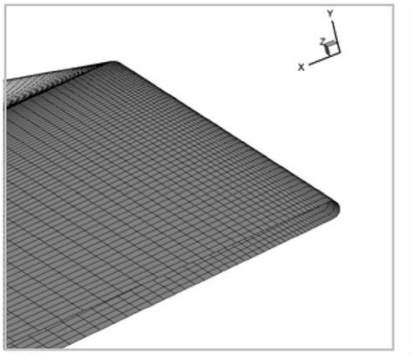

(c) Surface mesh of the inlet lip

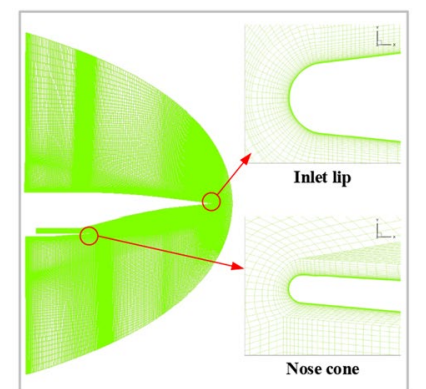

(b) Mesh near the wall

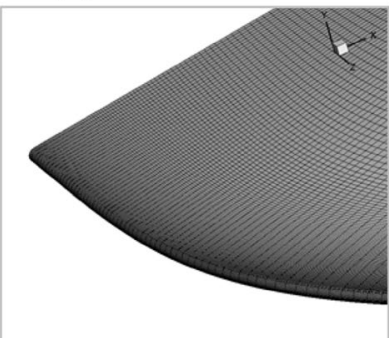

(d) Surface mesh of the nose cone
Fig. 5 Computational mesh and boundary condition settings

Table 1 Freestream and flight conditions of the study case

\begin{tabular}{lllll}
\hline Material & Mach number & Attack angle $\left(^{\circ}\right)$ & $P_{\infty}(\mathrm{Pa})$ & $T_{\infty}(\mathrm{K})$ \\
\hline Air & 4.5 & 0 & 5529 & 217 \\
\hline
\end{tabular}

the validation case are listed in Table 1 . The results show that the flux distribution and magnitudes appear as nearly constant (the maximum flux changes slightly from 1800 to $1785 \mathrm{~kW} / \mathrm{m}^{2}$ ) when the first-cell spacing refines from 0.01 to $0.005 \mathrm{~mm}$. Considering that the mesh amount should be reduced as much as possible to ensure computational efficiency under the precondition of guaranteeing precision, the spacing is chosen to be $0.01 \mathrm{~mm}$ in this case, as shown in Fig. 5b, such that the volume mesh number of the flow field domain is $17,619,000$. Furthermore, considering that either the nose cone or the inlet lip is a high heat flow region with a radius of $2 \mathrm{~mm}$, the grid cell dimension in these regions is set to be $0.2 \mathrm{~mm}$, as shown in Fig. $5 \mathrm{c}$, d.

\subsection{FEM Model}

\subsubsection{Governing Equations}

The basic governing equation of the three-dimensional structural heat transfer is the classical heat conduction equation that can be expressed as:

$\rho_{\mathrm{s}} c_{\mathrm{s}} \frac{\partial T_{\mathrm{s}}}{\partial t}=\frac{\partial}{\partial x_{i}}\left(k_{\mathrm{s}} \frac{\partial T_{\mathrm{s}}}{\partial t}\right)+Q \quad(i=1,2,3)$, where $T_{\mathrm{s}}$ is the structural temperature, $\rho_{\mathrm{s}}$ is the structural density, $c_{\mathrm{s}}$ is the structural specific heat, $k_{\mathrm{s}}$ is the structural thermal conductivity, and $Q$ is the volumetric heating source in a solid.

\subsubsection{Numerical Discretization}

In this study, the FEM is used to discretize the above transient solid heat conduction equation. Then the classical discretized FEM matrix formulation can be rewritten as:

$[\boldsymbol{C}]\{\dot{\boldsymbol{T}}\}+[\boldsymbol{K}]\{\boldsymbol{T}\}=\{\boldsymbol{Q}\}$.

Here, $[\boldsymbol{C}],\{\boldsymbol{T}\},[\boldsymbol{K}],\{\boldsymbol{Q}\}$ denote the thermal capacitance matrix, temperature vector, thermal conductivity matrix, and nodal heat load vector, respectively.

\subsubsection{FEM Model for Hypersonic Flight}

In this work, the numerical solutions of Eq. (22) were obtained using the general finite element solver ABAQUS Version 6.13 programmer [1], and the unconditional stable backward Euler method [6] is adopted to march the solution in time. The FEM computational grid is an unstructured grid generated by ABAQUS, as illustrated in Fig. 6, and the analysis element type is set as DC3D20. Then, the volume mesh number of the domain is 300,913 .

Three boundary conditions are applied in the model: (1) The aerodynamic heat flux on the interfacial surface which is calculated by Polysim; (2) The radiation boundary condition on the external surface that describes the radiative heat exchange with the surroundings; (3) Adiabatic boundary conditions on the other surfaces. Furthermore, three kinds of materials are utilized in different regions. High temperature alloy is utilized in the nose cone region, high temperature insulation composite material is utilized in the cabin region, and the $\mathrm{C} / \mathrm{C}$ composite material is utilized in the inlet region.

\subsection{Interface Coupling Relationship Model}

The physical mechanism of the coupled flow-thermal problem for the hypersonic vehicle is that the external hypersonic

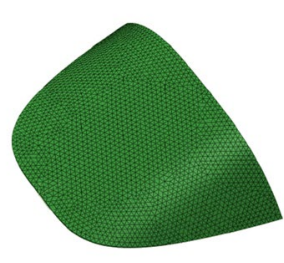

(a) The nose cone region

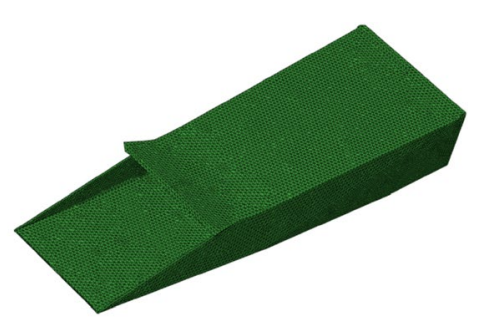

(b) The inlet region
Fig. 6 Computational mesh of FEM model 
aerodynamic heating interacts with the structural heat transfer inside the solid via a fluid-solid interface, along which the temperature continuity condition [23] and the heat flux equilibrium condition [24] must satisfy

$T_{\mathrm{s}}=T_{\mathrm{f}}$,

$k_{\mathrm{s}} \nabla T_{\mathrm{s}} \cdot \boldsymbol{n}_{\mathrm{s}}=-k_{\mathrm{f}} \nabla T_{\mathrm{f}} \cdot \boldsymbol{n}_{\mathrm{f}}$.

Here, $T_{s}$ and $T_{\mathrm{f}}$ represent the structural and fluid temperature, respectively; $\boldsymbol{n}_{s}$ and $\boldsymbol{n}_{\mathrm{f}}$ represent the outward unit normal of the structural and fluid boundaries, respectively; and $k_{\mathrm{s}}$ and $T_{\mathrm{f}}$ are the conductivities of the structure and fluid, respectively.

As concluded by Giles [8], the numerical stability could be improved by passing temperature from the solid to the fluid while transferring heat flux from the fluid to the solid. Thus, in this study, the thermal solver ABAQUS provides temperature to the fluid solver Polysim, whereas heat flux is transferred from the Polysim to the ABAQUS in the form of the surface heat transfer coefficient. The interpolations of temperature and the surface heat transfer coefficient are conducted with the inverse-distance interpolation algorithm provided in Tecplot 360 EX 2015 R1 [31]. The inverse-distance interpolation averages the values at the data points from the source zone to the data points in the destination zone. The average is weighted by a function of the distance between each source data point to the destination data point. The closer a source data point is to the destination data point, the greater its value is weighted.

\section{Results and Discussions}

\subsection{Trajectory Profile}

For this paper, the flight conditions including the flight altitude $(H)$, the Mach number $(M a)$ and the attack angle

Table 2 Flight conditions for all the trajectory points

\begin{tabular}{lrllr}
\hline No. & $t(\mathrm{~s})$ & $h(\mathrm{~km})$ & $M a(-)$ & $\alpha\left(^{\circ}\right)$ \\
\hline 1 & 0 & 10 & 2 & -2 \\
2 & 20 & 15 & 4 & 0 \\
3 & 35 & 25 & 6.5 & 0 \\
4 & 50 & 35 & 6.5 & -1 \\
5 & 70 & 45 & 6.5 & -1 \\
6 & 120 & 50 & 5 & 4 \\
7 & 175 & 35 & 6 & 4 \\
8 & 190 & 25 & 6 & 1 \\
9 & 200 & 20 & 6 & 1 \\
10 & 220 & 25 & 6 & 0 \\
\hline
\end{tabular}

$(\alpha)$ along the trajectory time $(t)$ are given in Table 2. Note that, as the linear interpolation algorithm is utilized in the proposed methods, the flight trajectory profile can be shown graphically in Fig. 7.

\subsection{Validation of a Numerical Model Based on Wind Tunnel Tests}

To validate the in-house CFD code Polysim, wind tunnel tests of a 1:2 scaled air-breathing hypersonic flight model is conducted. The model is tested under the conditions of Mach 6, unit Reynolds number $1.14 \times 10^{7}$ and attack angles $0^{\circ}, 2^{\circ}, 4^{\circ}$.

As shown in Fig. 8, the results can be summarized as follows: (1) Primarily, the heat flux on the forebody front edge stagnation point is compared. In the wind tunnel test, the measured value of the heat flux on the front edge stagnation point is $1050 \mathrm{~kW} / \mathrm{m}^{2}$ while the calculated value obtained by Polysim is $1047 \mathrm{~kW} / \mathrm{m}^{2}$. It can be seen that the test value in the wind tunnel and the simulation value calculated by Polysim of the heat flux at the front edge stagnation point are close. (2) Furthermore, considering the complex flow around the inlet lip, the comparisons of the heat flux along the lip are illustrated in Fig. 8, where the heat flux in the stagnation point is selected as the reference value. It indicates that the maximum error between the test value and the corresponding simulation value is less than 15\%. (3) Eventually, the tendencies of the heat flux along the inlet lip between the wind tunnel test and the numerical simulation are consistent under different attack angles. Therefore, it can be concluded that the in-house CFD code Polysim is effective and adoptable in this work.

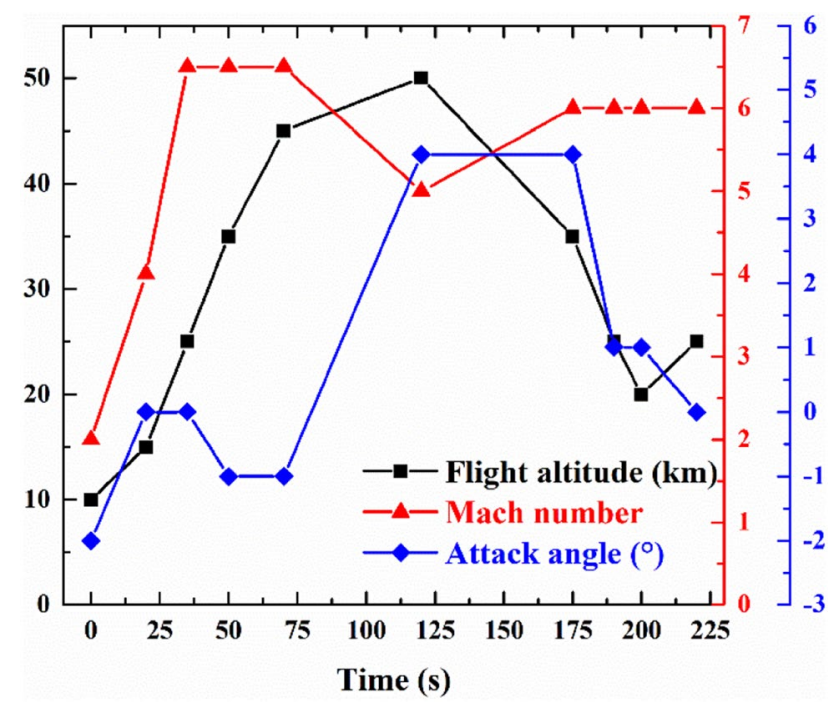

Fig. 7 Flight trajectory profile 

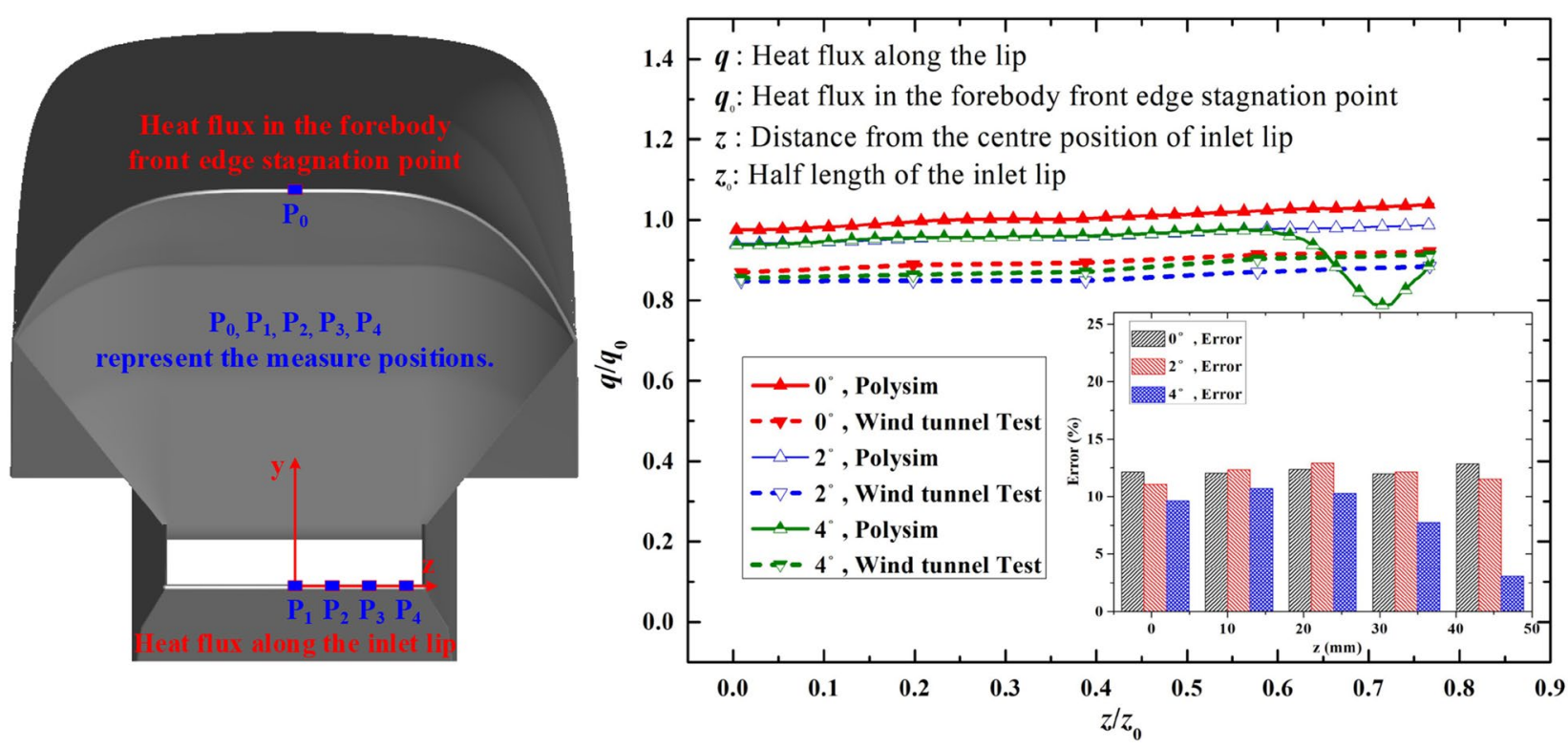

Fig. 8 Comparisons of heat flux along the lip between wind tunnel tests and numerical calculations

\subsection{Aerodynamic Heat Analysis Based on Polysim}

Prior to conduction flow-thermal interaction simulations, the steady-state flowsfields around the hypersonic vehicles are obtained. For the complex configuration of the air-breathing hypersonic vehicle, complicated flow patterns and different forms of flow interference arise. The intense shock wave interference effect leads to the severe aerodynamic heating environment. Figure 9 shows the flow structure of a typical trajectory point at which $h=25 \mathrm{~km}, M a=6.5, \alpha=0^{\circ}$. The following can be determined:

1. As displayed in Fig. 9 (on the left) for the overall density distribution, influenced by the windward configuration, two extra compression waves are induced, namely, the two-stage and the three-stage compression waves. Furthermore, as shown in the enlarged view of region (a) in Fig. 9 for pressure distribution, a smooth bow shock wave appears around the arc-shaped leading edge.

2. As shown in the enlarged view of region (b) for pressure distribution, a smooth bow shock wave appears around the arc-shaped inlet lip similar to at the leading edge. Moreover, the incident shock wave caused by the leading edge and the shock wave caused by the inlet lip interfere strongly with each other around the lip. Thus, the local separation flow phenomenon may be triggered.

3. As illustrated in the enlarged view of region (c) for density distribution, owing to the interference effect of the shock waves caused by the leading edge, the compression surface and the inlet lip as well as the reflection
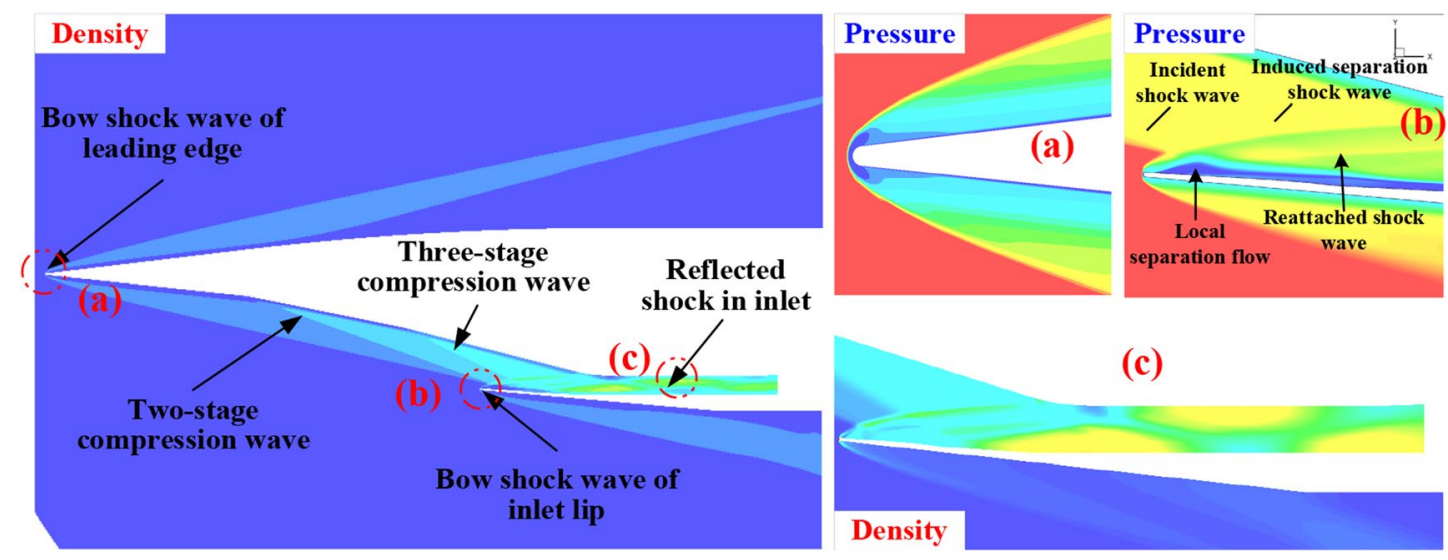

Fig. 9 Flow structure characteristics. On the left, overall view; on the right, zoomed regions 
effect of the shock waves in the isolator, the shock train appears in the inlet isolator, thus the high and low heat flow alternatively present in either the upper or the lower wall of inlet.

Figure 10 illustrates the heat flux distribution at typical trajectory points. Figure 10a shows the heat flux along the inlet lip plotted against the $z$-coordinate. One can see that heat flux values are large for the shock wave effect and vary inconspicuously along the lip for the smooth configuration. Figure 10b, c show the heat flux along the upper surface and lower surface of the compression surface, respectively, plotted against the $x$-coordinate. The heat flux demonstrates a ladder-like shape, which is in accordance to the three stages of compression waves shown in Fig. 9. Further, the heat flux in the isolator varies with a wave-shape due to the reflection effect of the shock waves.

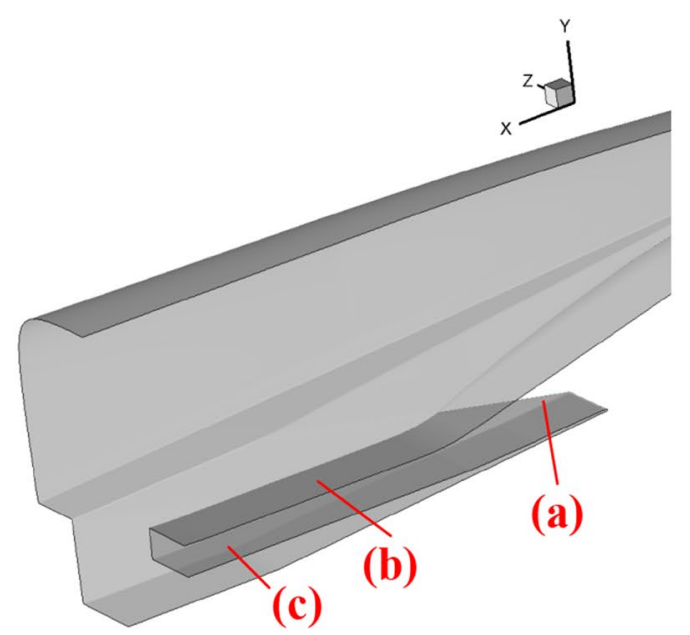

Illustration of typical positions

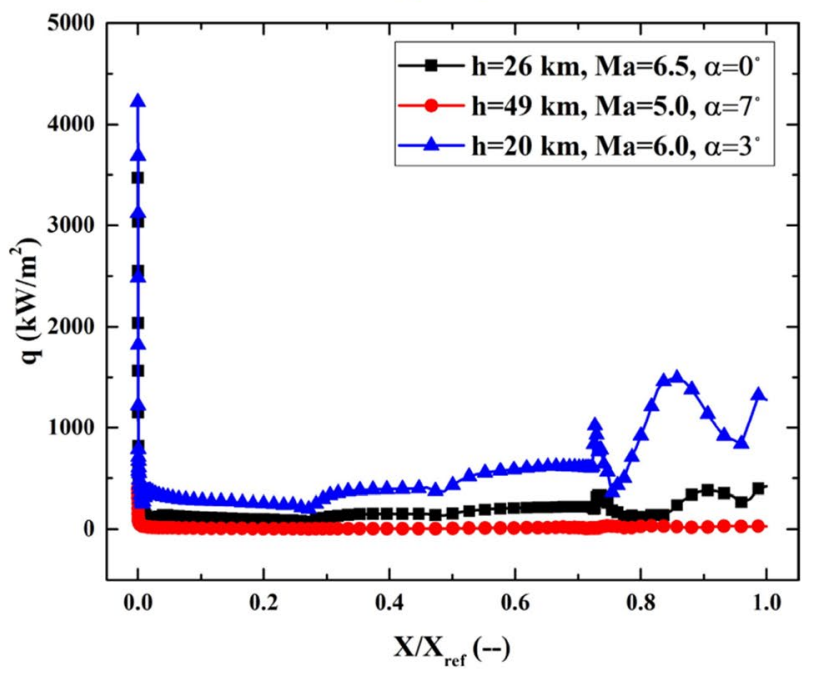

(b) Heat flux along the upper surface of the compression surface and inlet

\subsection{Comparisons of BDCM and IDCM}

Figure 11 compares the thermal responses of the TPS calculated using BDCM and IDCM. The maximum temperature history of all structural parts and the temperature history of a specific point around the stagnation point of the nose cone are selected. Figure 11a shows that both the BDCM and IDCM exhibit a similar tendency, which reflects the consistency of the two methods to a certain extent. However, as illustrated in Fig. 11b, the BDCM ignores the change in the recovery temperature and the cold wall heat flux between the two trajectory points, which would lead to the non-smooth phenomenon of the temperature responses. Moreover, this simplification also leads to an overestimation of the highest temperature and underestimation of the lowest temperature. The IDCM considers the continuous change process of the recovery temperature and the cold wall heat flux by linear

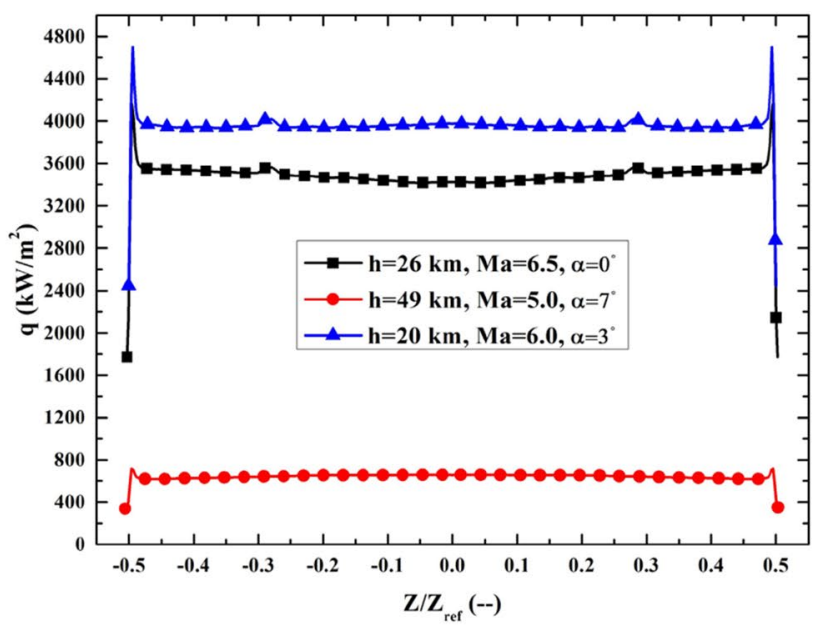

(a) Heat flux along the inlet lip

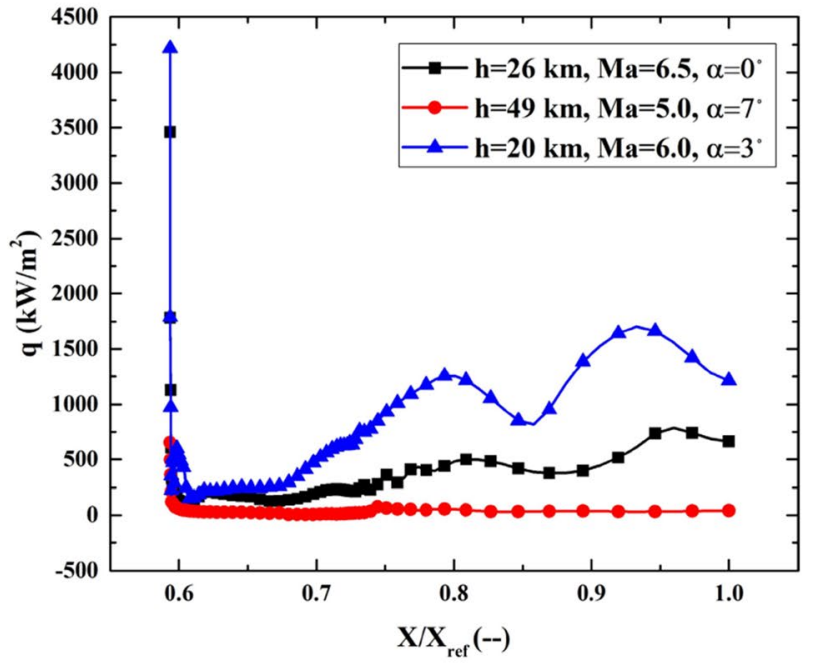

(c) Heat flux along the lower surface of the inlet

Fig. 10 Heat flux on typical positions for typical trajectory points 


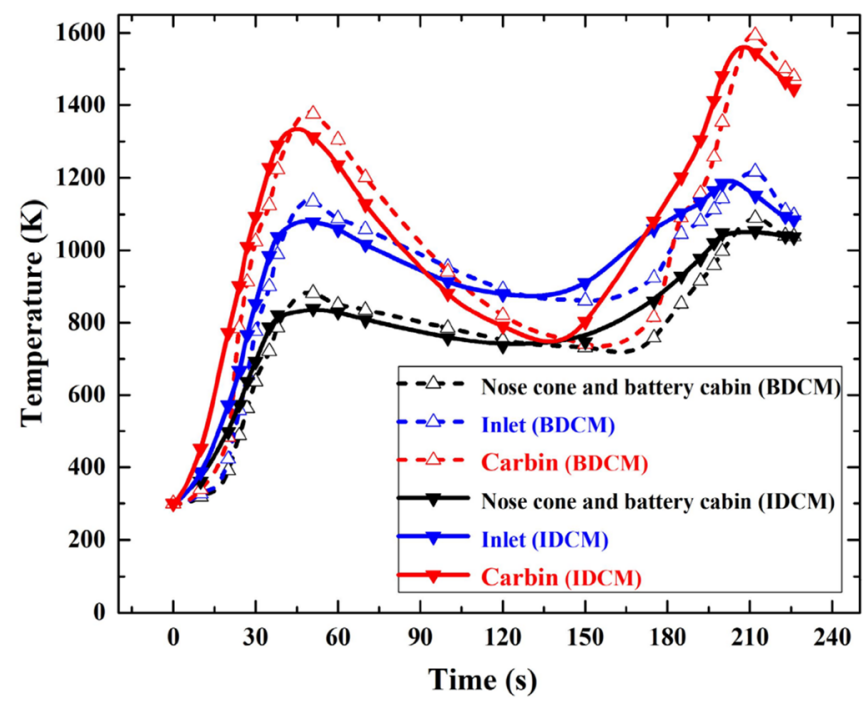

(a) Maximum temperature history of different components

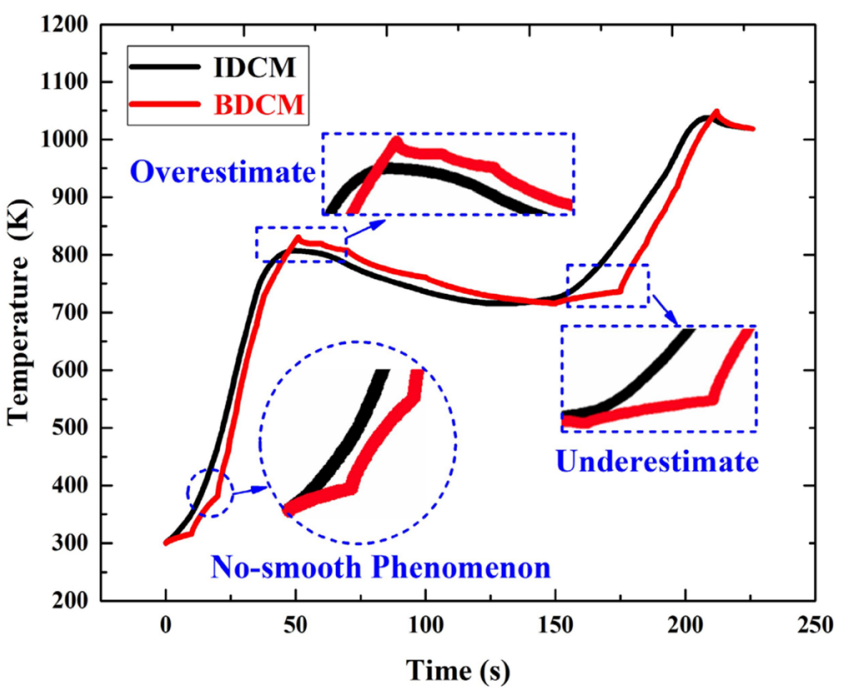

(b) temperature history of a specific point on the inlet

Fig. 11 Comparison of the thermal responses for BDCM and IDCM

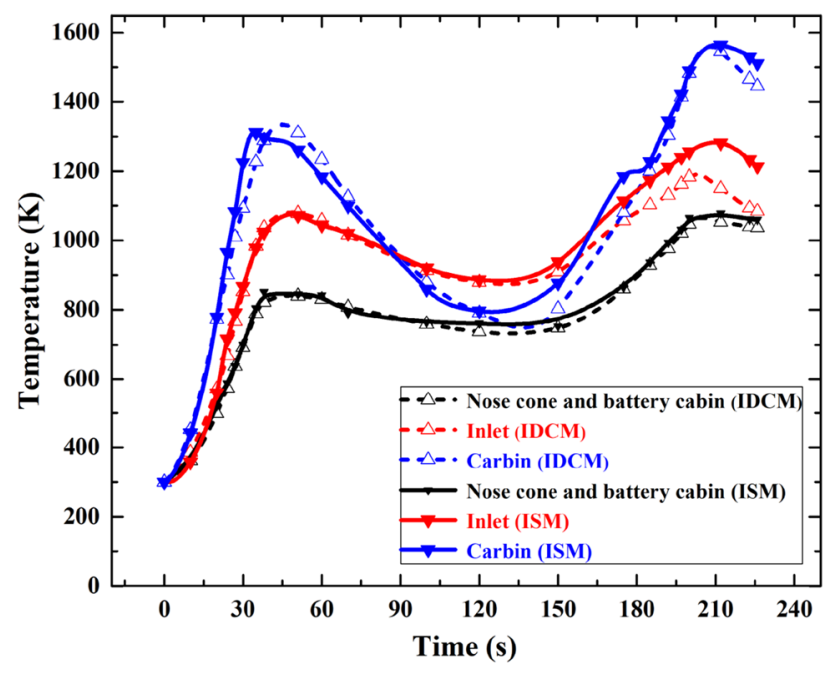

Fig. 12 Comparison of the maximum temperature history for different components under IDCM and ISM

interpolation between two trajectory points, which is much closer to the practical flight situation than that obtained using BDCM.

\subsection{Comparisons of IDCM and ISM}

Figures 12 and 13 show the comparison of the thermal responses of TPS calculated using IDCM and ISM. As illustrated in Fig. 12, IDCM and ISM demonstrate a similar tendency on the maximum temperature qualitatively, and the maximum temperature obtained in IDCM is similar to ISM quantitatively. As displayed in Fig. 13, the maximum temperatures of both IDCM and ISM are located at the leading edge stagnation point. Moreover, the temperature obtained from IDCM is higher than that from ISM in nonstagnation point regions.

It is well known that the temperature distribution depends on the heat flux distribution. For IDCM, the heat flux conducted into the TPS is the corrected cold wall heat flux, which can be calculated by Eq. (5). For ISM, the heat flux conducted into the TPS is the hot wall heat flux, which can be calculated by the CFD analysis under the actual wall temperature distribution. As for the maximum temperatures of both IDCM and ISM, they are located at the leading edge stagnation point since the heat flux is greatest there. Furthermore, for IDCM, the recovery temperature $T_{\mathrm{r}}$ can be accurately estimated by Eq. (9). Thus, the heat flux at leading edge stagnation point obtained by IDCM is accurate enough. For ISM, it takes a full consideration of the coupling effect between the fluid and structure by CFD analysis under the actual wall temperature distribution. Thus, ISM can obtain the accurate heat flux at the stagnation point. Therefore, it can be concluded that IDCM and ISM can acquire the similar heat flux at stagnation point, which can be also demonstrated by the corrected cold wall heat flux and hot wall flux in Fig. 14. Moreover, the maximum temperature obtained by IDCM and ISM are similar due to the similar heat flux.

In the non-stagnation point region, the recovery temperature $T_{\mathrm{r}}$ estimated by Eq. (9) is relatively larger than the accurate recovery temperature in IDCM while the recovery temperature is still accurate by utilizing CFD analysis under the actual wall temperature distribution in ISM. Therefore, according to Eq. (5), the calculated heat flux in IDCM is larger than that in ISM, which results in the phenomenon 
Fig. 13 Comparison of the temperature distributions obtained by IDCM and ISM
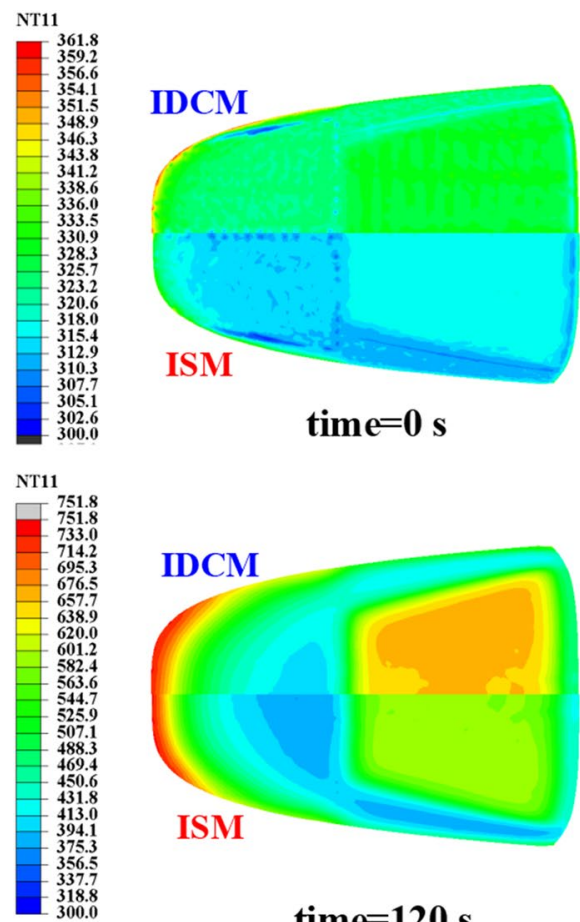

time $=\mathbf{0} s$

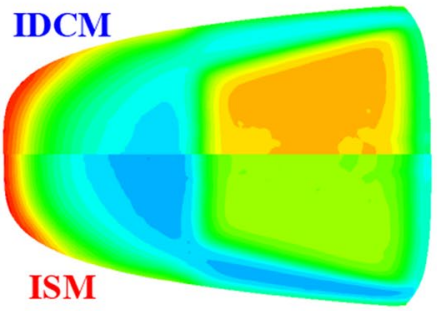

time $=120 \mathrm{~s}$
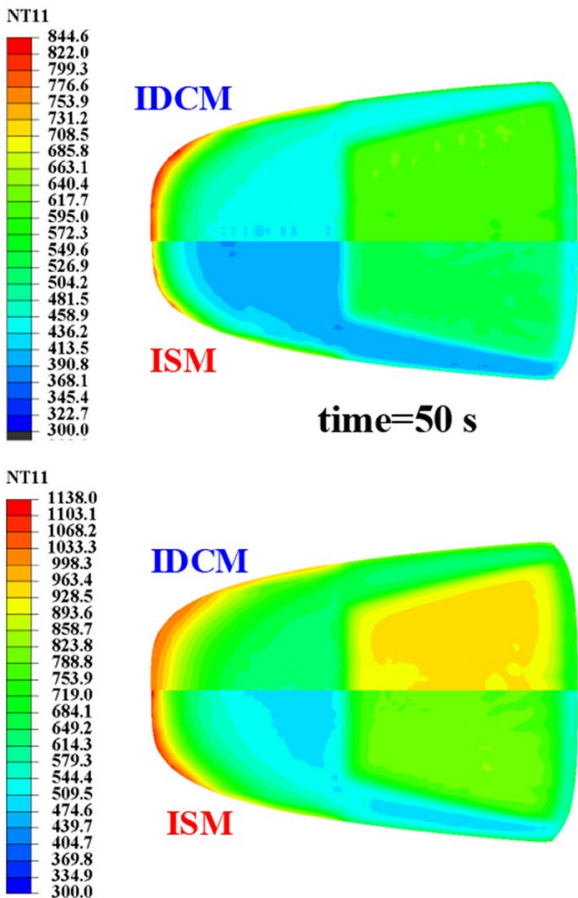

time $=50 \mathrm{~s}$

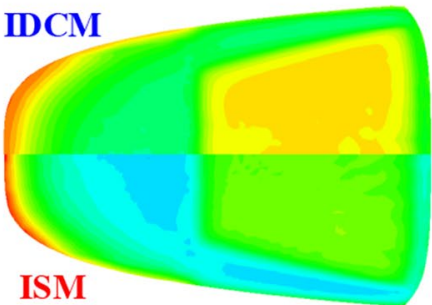

time $=\mathbf{2 0 0} s$

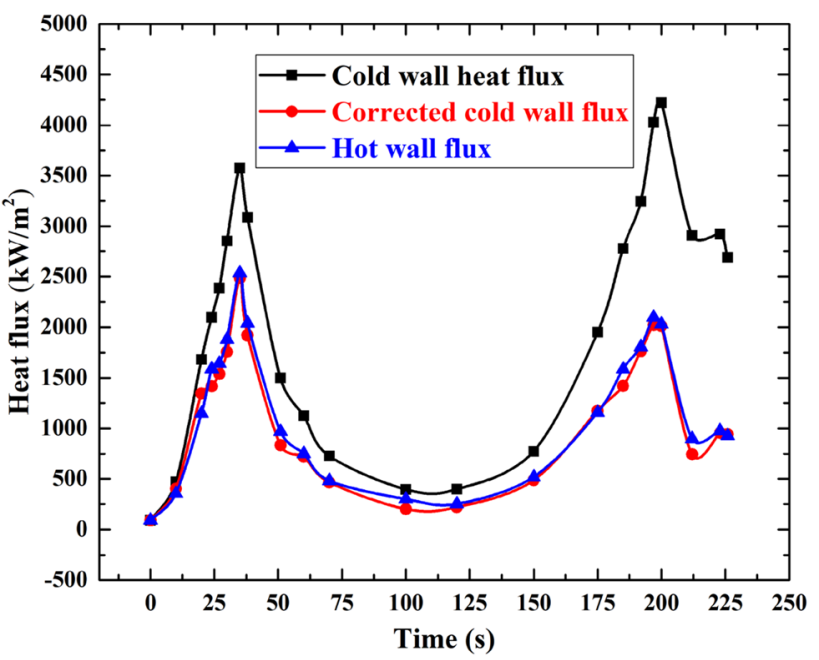

Fig. 14 Comparison of the heat flux at the front edge stagnation point

that the temperature obtained from IDCM is higher than that from ISM in most regions.

Additionally, for the computational accuracy, ISM takes a full consideration of the coupling effect between the fluid and structure; therefore, more accurate results can be obtained. However, for the computational efficiency, IDCM exploits a full advantage of the parallel computation strategy.

To explore the influence of the wall temperature distribution on the aerodynamic characteristics, the heat flux values at the stagnation point between the cold wall and hot wall are compared in Fig. 14. The heat flux values agree in terms of the trend over the trajectory under the conditions of cold wall and hot wall. The heat flux decreases sharply under the hot wall situation because the difference between the recovery temperature and wall temperature under the hot wall is much smaller than that under the cold wall, and the heat flux is proportional to the difference according to Eq. (4).

The comparisons of the surface heat flux and heat transfer coefficient between the cold wall and hot wall are shown in Fig. 15. The amplitude of the surface heat flux under the hot wall is much smaller than that under the cold wall, whereas the heat transfer coefficients in these two conditions are very close. This finding indicates that the surface heat flux is strongly coupled with wall temperature, whereas the heat transfer coefficient is weakly coupled with the wall temperature.

To determine the characteristics of the local flow field under different wall temperatures, the Mach contour in the inlet between cold wall and hot wall is compared in Fig. 16. A notably thickened boundary layer is observed in the hot wall calculation. Furthermore, due to the closed and limited space inside the inlet, the flow interference effect is relatively strong, and the internal flow structure is complicated with a hot wall calculation. The thickened boundary layer may change the aerodynamic force and intensify the phenomenon of flow separation. Together with the complicated internal flow structure, these changes could have a major impact on the flight performance, such as engine starting performance. 


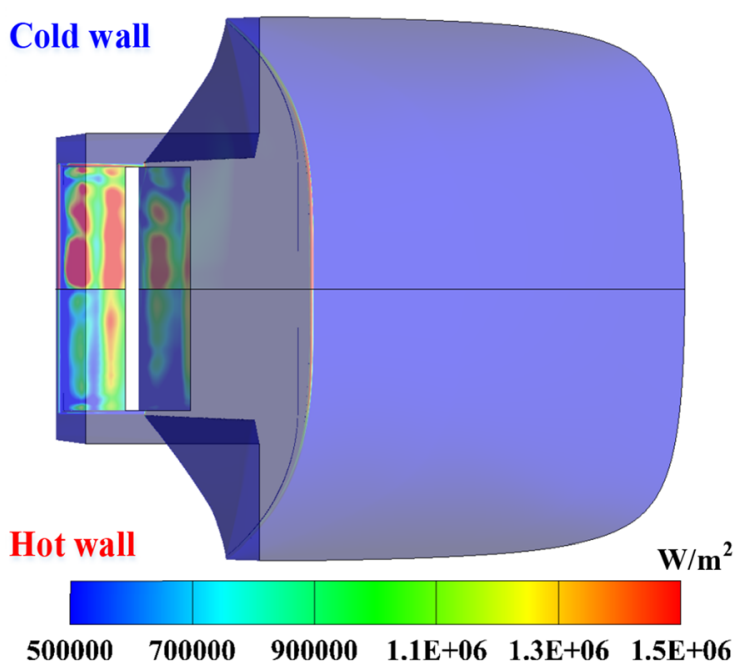

(a) The surface heat flux distribution

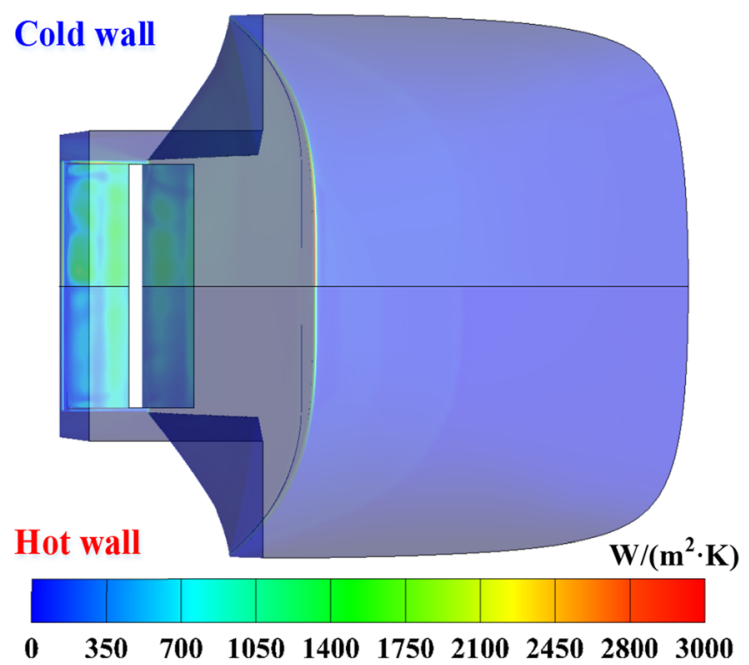

(b) The surface heat exchange coefficient distribution

Fig. 15 Comparison of the surface heat flux and heat transfer coefficient distribution when $t=230 \mathrm{~s}$

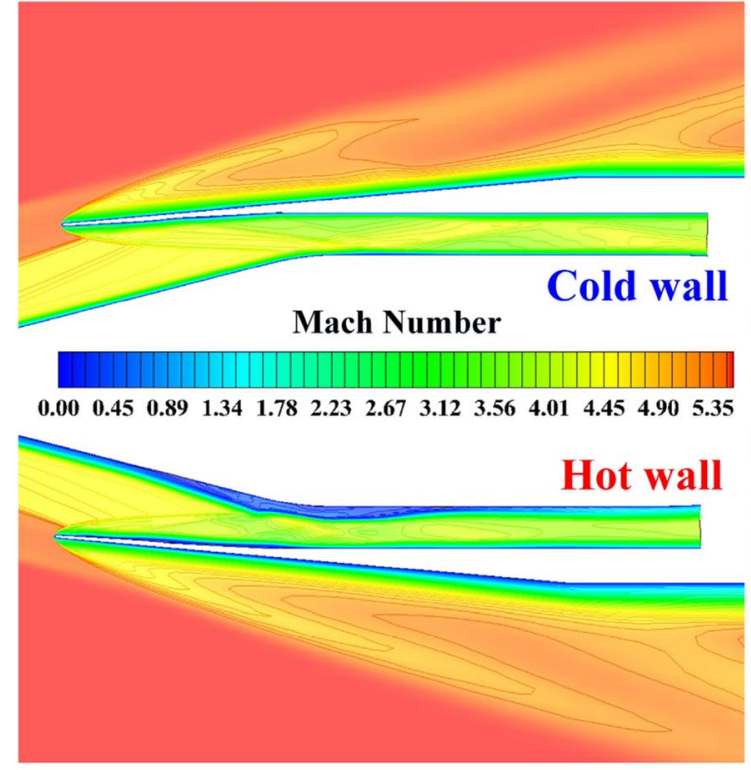

$\mathbf{t}=\mathbf{1 2 0} \mathrm{s}$

Fig. 16 Comparison of the inlet flow structure

\subsection{Discussions of Precision and Efficiency}

According to the aforementioned cases and comparisons, the following can be summarized:

1. The tendencies of the maximum temperature values of all the three components for the air-breathing vehicle are consistent in DCMs and ISM. However, for DCMs, the impact of the hot wall on the flow characteristics is ignored, which means that some physical phenomena

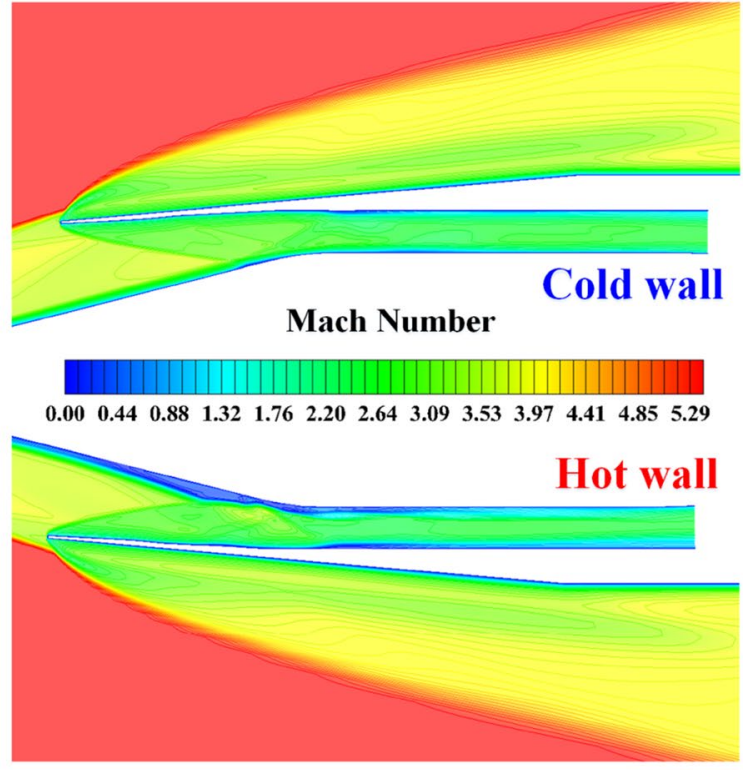

$\mathbf{t}=\mathbf{2 0 0 s}$ cannot be captured. The ISM however, considered the effects of the hot wall, and real physical processes such as thickened boundary layer and flow separation could be reproduced. Therefore, in terms of the simulation precision, ISM has an advantage over DCMs.

2. As the CFD analysis consumes much more time than the FEM analysis, in this work, the runtime is approximated as the CFD analyses time. As for the computational efficiency, on the one hand, the runtime is dependent on the iteration number. For DCMs, only one CFD analysis 
Table 3 Testing environment and computational efficiency evaluation of IDCM and ISM

\begin{tabular}{|c|c|c|c|c|c|}
\hline \multirow[t]{2}{*}{ Hardware environment } & \multirow[t]{2}{*}{ Software environment } & \multirow{2}{*}{$\begin{array}{l}\text { Number } \\
\text { of CFD } \\
\text { analyses }\end{array}$} & \multirow[t]{2}{*}{ Multicomputer system } & \multicolumn{2}{|c|}{ Runtimes (h) } \\
\hline & & & & DCMs & ISM \\
\hline CPU:2.40 GHz & System: Windows 7 & 10 & Unavailable & 576 & 1104 \\
\hline $\begin{array}{l}\text { Memory: } 192 \mathrm{~GB} \\
\text { Number of processors: } 44\end{array}$ & $\begin{array}{l}\text { Program: Polysim } 1.01 \\
\text { ABAQUS } 6.13\end{array}$ & 28 & Available & 72 & 1104 \\
\hline
\end{tabular}

is needed in every trajectory interval; however, more than two iterations are required for ISM to reach convergence in every trajectory interval. In this work, based on the defined convergence criterion, three iterations are needed in every trajectory interval for the hypersonic vehicle case. Therefore, the iteration number of the DCM is 10 while that of ISM is 28 . On the other hand, the runtime is also dependent on the time that each CFD analysis consumes as the adjustable strategy stated in Sect. 3.3 is conducted in ISM. Therefore, the runtime for DCM and ISM is also given. Moreover, the parallel strategy can be adopted in DCMs, but only the sequential strategy can be utilized in ISM. The parallel strategy utilized in DCMs can be explained as follows: steady hypersonic flow simulations of all the discrete flight conditions under the cold wall condition should be obtained for DCMs. Since the simulations of all the discrete trajectory points are generally independent of each other, these can be conducted simultaneously. Therefore, when the multicomputer system is available, the CFD analyses can be executed by parallel strategy and the computational time can be substantially reduced. Consequently, for the computational efficiency, DCMs have a prominent advantage over ISM, in particular, in situations in which the multicomputer system is available, and the parallel strategy can be executed in DCMs. To compare the computational efficiency of DCMs and ISM, the runtimes for the fluid-thermal analysis of the air-breathing hypersonic flight are quantified, as listed in Table 3, which also summarizes the testing environment.

3. Given the above, in the practical process of hypersonic vehicle design and manufacture, we should select the appropriate analysis method according to different stages. In the conceptual design stage, the shape and structure form of the flight keep updating; in this case, a high-efficiency analysis method is adopted to help the designer make a quick and preliminary decision, and thus, DCM is a good choice. In the detailed design stage, accurate flight performance is necessary, and thus, the influence of hot wall on the flow field cannot be neglected. Therefore, ISM is a better choice to obtain a more reliable design scheme.

\section{Conclusions}

The study of the coupling flow-thermal problem is particularly important for the design and evaluation of the thermal protection system for air-breathing hypersonic vehicles. In this paper, two kinds of coupled analysis strategies, i.e., DCMs and ISM, are presented and compared. Both strategies are achieved through a finite-element thermal response code and a self-compiled finite-volume flow code, the accuracy of which is verified by the wind tunnel test.

Both DCMs and ISM can acquire rational results. However, each of the two strategies has its own advantages and disadvantages. For the simulation precision, ISM has an advantage over DCMs since the real flow characteristics can be captured. However, for the computational efficiency, DCMs have a remarkable advantage over ISM. In view of this, a preliminary selection criterion is established in this paper, and then in the actual process of air-breathing hypersonic vehicle design and manufacture, the designers can select the appropriate flow-thermal analysis method according to the different design stages.

Acknowledgements Financial supports from National Natural Science Foundation of China (Grant Nos. 11902322, 51602348) and National Defense Basic Scientific Research program of China (No. JCKY2016130B009) are gratefully acknowledged.

\section{References}

1. ABAQUS Documentation (2014) ABAQUS Documentation, Version 6.13. Simulia Corp., Providence

2. Bilal AB, Clark HL (1992) New technique for low-to-high altitude predictions of ablative hypersonic flowfields. J Spacecr Rockets 29(1):35-50

3. Chen YK, Henline WD (1993) Chemical nonequilibrium NavierStokes solutions for hypersonic flow over an ablating graphite nosetip. In: AIAA 28th Thermophysics Conf., AIAA

4. Chen YK, Henline WD, Tauber ME (1995) Mars pathfinder trajectory based heating and ablation calculations. J Spacecr Rockets 32(2):225-230

5. Conti RJ, MacCormack RW, Groener LS, Fryer JM (1992) Practical navier-stokes computation of axisymmctric reentry flowfields 
with coupled ablation and shape change. In: 30th aerospace sciences meeting \& exhibit, AIAA

6. Cook D, Malkus DS, Plesha M (1974) Concepts and applications of finite element analysis. Wiley, New York, pp 484-486

7. Ferrero P, D'Ambrosio D (2008) A numerical method for conjugate heat transfer problems in hypersonic flows. In: AIAA 40th thermophysics conf., AIAA

8. Giles MB (1997) Stability analysis of numerical interface conditions in fluid-structure thermal analysis. Int J Numer Methods Fluids 25(4):421-436

9. Gori F, Corasaniti S, Worek WM, Minkowycz WJ (2012) Theoretical prediction of thermal conductivity for thermal protection systems. Appl Therm Eng 49:124-130

10. Guo S, Xu J, Qin Q, Gu R (2016) Fluid-thermal interaction investigation of spiked blunt bodies at hypersonic flight condition. J Spacecr Rockets 53(4):1-15

11. Gupta RN, Lee KP, Moss JN, Sutton K (1992) Viscous shock-layer solutions with coupled radiation and ablation for earth entry. J Spacecr Rockets 29(2):173-181

12. Hassan B, Kuntz D, Potter D (2013) Coupled fluid/thermal prediction of ablating hypersonic vehicles. In: AIAA aerospace sciences meeting and exhibit, AIAA

13. Henline WD (1992) Thermal protection analysis of Mars-earth return vehicles. J Spacecr Rockets 29(2):198-207

14. Koerber S (1999) Fluid-structure-interaction focused on aspects of aerokinetic heating. In: 30th fluid dynamics conference, AIAA

15. Kontinos D (2012) Coupled thermal analysis method with application to metallic thermal protection panels. Acta Neurol Belg 11(2):173-181

16. Kuntz DW, Hassan B, Potter DL (1999) An iterative approach for coupling fluid/thermal predictions of ablating hypersonic vehicles. In: AIAA 33nd thermophysics conf., AIAA

17. Kuntz DW, Hassan B, Potter DL (2001) Predictions of ablating hypersonic vehicles using an iterative coupled fluid/thermal approach. J Thermophys Heat Transf 15(2):129-139

18. Ma Y, Xu B, Chen M, He R, Wen W, Cheng T, Fang D (2017) Optimization design of built-up thermal protection system based on validation of corrugated core homogenization. Appl Therm Eng 115:491-500

19. Marín E (2010) Characteristic dimensions for heat transfer. Latin Am J Phys Educ 4(1):56-60

20. Mcnamara JJ, Friedmann PP (2007) Aeroelastic and aero-thermoelastic analysis of hypersonic vehicles: current status and future trends. In: 48th AIAA/ASME/ASCE/AHS/ASC structures, structural dynamics, and materials conf., AIAA, Reston

21. Meng F, Dong S, Peng L, Wang J (2016) A new algorithm of global tightly-coupled transient heat transfer based on quasisteady flow to the conjugate heat transfer problem. Theor Appl Mech Lett 6(5):233-235

22. Olynick DR, Chen YK, Tauber ME (1997a) Forebody TPS sizing with radiation and ablation for the stardust sample return capsule. In: AIAA 32nd thermophysics conf., AIAA

23. Olynick DR, Chen YK, Tauber ME (1997b) Wake flow calculations with ablation for the stardust sample return capsule. In: AIAA 32nd thermophysics conf., AIAA

24. Pointwise User Manual (2016) Pointwise User Manual, Version 18.0, Pointwise Inc. Fort Worth

25. Poteet C, Abu-Khajeel H, Hsu SY (2002) Preliminary thermalmechanical sizing of metallic TPS-process development and sensitivity studies. In: 40th aerospace sciences meetings and exhibitions
26. Qin Q, Xu J, Guo S (2017) Fluid-thermal analysis of aerodynamic heating over spiked blunt body configurations. Acta Astronaut 132:230-242

27. Rahaim CP, Kassab AJ, Cavalleri RJ (2015) Coupled dual reciprocity boundary element/finite volume method for transient conjugate heat transfer. J Thermophys Heat Transf 14(1):27-38

28. Sudalagunta PR, Sultan C, Kapania RK, Watson LT, Raj P (2015) Aeroelastic control-oriented modeling of an air-breathing hypersonic vehicle. In: Dynamics specialists conf., pp 1-14

29. Sun J, Zhang G, Vlahopoulos N, Hong SB (2006) Multi-disciplinary design optimization under uncertainty for thermal protection system applications. In: 11th AIAA/ISSMO multidisciplinary analysis and optimization conf., AIAA

30. Tabiei A, Sockalingam S (2012) Multiphysics coupled fluid/ thermal/structural simulation for hypersonic reentry vehicles. J Aerospace Eng 25(2):273-281

31. Tecplot Help Viewer (2015) Tecplot 360 Help Viewer, Version 2015 R1, Tecplot, Bellevue

32. Thornton EA, Dechaumphai P (1988) Coupled flow, thermal, and structural analysis of aerodynamically heated panels. J Aircr 25(11):1052-1059

33. Weiss M, Smith WA (1995) Preconditioning applied to variable and constant density flow. AIAA J 33:2050-2057

34. Witeof ZD, Neergaard LJ, Vanderwyst AS, Pasiliao C (2016) Dynamic fluid-thermal-structural interaction effects in preliminary design of high speed vehicles. In: 15th dynamics specialists conf., AIAA, Reston

35. Yan W, Luo X, Cui D (2015) Strength analysis and optimization technique for thermal protection system using 3 dimensional element model. In: AIAA modeling and simulation technologies conf., AIAA

36. Zhang S, Chen F, Liu H (2014) Time-adaptive, loosely coupled strategy for conjugate heat transfer problems in hypersonic flows. J Thermophys Heat Tranf 28(4):1-12

37. Zhao S, Li J, Zhang C, Zhang W, Lin X, He X, Yao Y (2014) Thermo-structural optimization of integrated thermal protection panels with one-layer and two-layer corrugated cores based on simulated annealing algorithm. Struct Multidiscip Optim 51(2):479-494

38. Zhao X, Meganathan A, Zhang S (2015) Computational approach for aeroheating with thermally coupled fields. J Thermophys Heat Transf 31(3):489-499

39. Zheng HW, Nicolleau FCGA, Qin N (2010) Assessment of DES on the flow after a snow-flake orifice. Notes Numer Fluid Mech Multidiscip Des 111:157-165

40. Zheng HW, Nicolleau FCGA, Qin N (2012) Detached eddy simulation of turbulence flows in a pipe with a snow flake fractal shape orifice. In: 8th Workshop on synthetic turbulence models

41. Zheng HW, Yang GW (2011) Investigation of aerodynamic performance of high-speed train by detached eddy simulation. In: Proceedings of 1st international workshop on high-speed and intercity railways, Iwhir

Publisher's Note Springer Nature remains neutral with regard to jurisdictional claims in published maps and institutional affiliations. 